\title{
Reduction in Hospitalisation for Cytomegalovirus Disease in HIV-Infected Patients Before and After the Introduction of Highly Active Antiretroviral Therapy
}

\author{
Holly Seale ${ }^{*}, 1$ D.E. Dwyer ${ }^{2}$ and C.R. MacIntyre ${ }^{3}$ \\ ${ }^{1}$ School of Public Health and Community Medicine, The University of New South Wales Sydney, NSW 2052, Australia \\ ${ }^{2}$ Centre for Infectious Diseases and Microbiology Laboratory Services, Institute of Clinical Pathology and Medical Re- \\ search (ICPMR), Westmead Hospital, New South Wales, Australia \\ ${ }^{3}$ National Centre for Immunization Research and Surveillance Preventable Disease, Children's Hospital at Westmead, \\ The University of Sydney, Australia
}

\begin{abstract}
Objective: The objective of this study was to describe trends in hospital admissions by HIV-infected patients for cytomegalovirus (CMV) disease in Australia in the eight year period from 1993 (before highly active antiretroviral therapy) to 2001 (widespread use of highly active antiretroviral therapy).

Study Design: Using data from the National Hospital Morbidity Database (NHMD) complied by the Australian Institute of Health and Welfare (AIHW), we examined the rates of hospital admissions for CMV in HIV-infected patients, by year, sex, age group, length of stay and number of diagnoses.

Results: Between 1993 and 2001, 21,846 patients were admitted with both HIV infection and CMV disease. $30.2 \%$ (6610/21846) of the HIV infected patients admitted to hospital were principally diagnosed with CMV disease. By 20002001 , the rate of hospital admissions was only 45 cases per $1000 \mathrm{HIV}$-infected population.

Conclusion: Dramatic decreases in the number of hospitalizations for CMV disease in HIV-infected patients have occurred over the eight-year study period in Australia, which may be related to the introduction of highly active antiretroviral therapy.
\end{abstract}

Keywords: Cytomegalovirus, HIV/AIDS, epidemiology, highly active antiretroviral therapy, hospitalization.

\section{INTRODUCTION}

Immunosuppression resulting from human immunodeficiency virus type 1 (HIV-1) infection leads to infection with various opportunistic agents that cause disease in their own right and can accelerate HIV disease progression [1]. Opportunistic infections (OIs) cause substantial morbidity, result in hospitalization, necessitate expensive and toxic therapies and shorten the survival of people with HIV infection [2-4].

Epidemiologic studies found that until 10 years ago, nearly one half of HIV-infected patients eventually developed CMV end-organ disease, including chorioretinitis, esophagitis, colitis, pneumonia, and central nervous system disease [5]. The spectrum of illness among people with AIDS has changed dramatically from advances in treatment and prophylaxis, and the availability of newer more potent antiretroviral drugs. The advent of highly active antiretroviral therapy (HAART) in 1996 made the crucial difference in AIDS-related OIs [6-8].

Since the introduction of HAART, a fivefold decrease in the incidence of CMV disease has been reported by several

\footnotetext{
*Address correspondence to this author at the School of Public Health and Community Medicine, The University of New South Wales Sydney, NSW 2052, Australia; Tel: +61 29385 3129; Fax: +61 29313 6185;

E-mail: h.seale@unsw.edu.au
}

authors [6, 9-12]. The current incidence of CMV disease in HIV patients is approximately 3-5 patient-years in the United States [6] and between 1 and 3.5 patient-years in France [13]. Moreover, the incidence of relapse in patients with previous history of CMV disease has also decreased dramatically [12]. For example, the incidence of CMV retinitis has decreased from 17.1 to 5.6 per 100 patient-years $[6,14]$. However, CMV retinitis remains the most frequent cause of visual loss in AIDS patients in developing countries [15].

Despite the obvious impact of HAART on the incidence of AIDS-related opportunistic infections [16], it is less clear whether it has also affected admissions rates for patients with an AIDS- associated disease. To determine the impact of HAART on the burden of patients with HIV and CMV, hospitalization data over the period 1993 to 2001, before and after the introduction of HAART were examined.

\section{METHODS}

A retrospective analysis of hospital discharge records from 1993 to 2001 was conducted. The data were drawn from the National Hospital Morbidity Database (NHMD) complied by the Australian Institute of Health and Welfare (AIHW) from data supplied by the State and Territory health authorities [17]. The NHMD is a collection of electronic confidentialised summary records for admitted patients dying/discharged from public and private acute and psychiatric 
hospitals, as well as private freestanding hospital facilities in Australia. Hospitalisations were chosen because they are more accessible in the database and less subject to interpretation than outpatient cases of CMV disease. Invasive CMV disease in HIV patients is most likely to be treated in hospitals, which makes the hospitalisation database a reasonable reflection of the true burden of HIV-associated CMV disease. The data were analyzed by financial year based on the date of hospital discharge. Discharge records include such information as discharge diagnoses and procedure codes, patient demographics (e.g. sex, age group, year and separation mode), and hospital attributes (average length of stay, number of diagnosis per hospitalisation).

We identified CMV/HIV hospitalisations by examining all primary and secondary discharge codes. Any admission with a specific HIV and CMV ICD 9 code: 042 and 0.78.5, 484.1 for the period 1993-1998, or ICD-10: B20-24 and B25, B27.1 for the period 1999-2001, were included. The introduction of HAART took place in late 1996; however the consequence of HAART in the change of incidence and prognosis of CMV disease would not have been expected to be detectable in the first few months of therapy.

To determine the denominator of people living with HIV, information from the National Centre for HIV Epidemiology and Clinical Research was used. The National Centre is responsible for coordinating the Australian National Surveillance for AIDS from statutory notifications by doctors, of AIDS and HIV diagnoses to the Department of Health in each State and Territory [18]. Notifications are made by pri- mary care and hospital based physicians. Estimations of the total population living with HIV were made using data obtained by the centre; these data was then used as the denominator when calculating the annual average rate of hospitalization. The denominator data was available by age and sex per financial year. All analysis was done using EPI-INFO (CDC, Version: 3.4.1, 2007) and Egret (Cytel Software, Version: 2.0.31, 1999). Statistical significance was defined as $\mathrm{p}<0.05$.

\section{RESULTS}

In Australia between 1993 and 2001, 21,845 patients were admitted with both HIV infection and CMV disease. In 1993-1994, the average rate of admissions was 102 cases per $1000 \mathrm{HIV}$-infected population. The rate then peaked from 1995-1996 and 1996-1997 at 914 cases per 1000 and 660 cases per 1000 HIV population respectively. These two periods combined equated for $70.1 \%$ of the admissions for the whole of the study period. After 1996-1997, the rate fell by $40.0 \%$ and by $2000-2001$, the rate of hospital admissions was only 44.9 cases per $1000 \mathrm{HIV}$-infected population (Fig. 1). A Chi-square test for trend indicated a significance association between CMV/HIV admissions and HIV population of Australia for the time period of 1993-1994 to1996-1997 $(\mathrm{n}=8156, \mathrm{p}=<0.05)$ and $1997-1998$ to $2000-2001 \quad(\mathrm{n}=2822$, $\mathrm{p}=<0.05)$.

Over the eight years, 140,011 bed days (average 17,501 days per year) were recorded for patients with CMV and HIV-infection. The median length of stay was one day

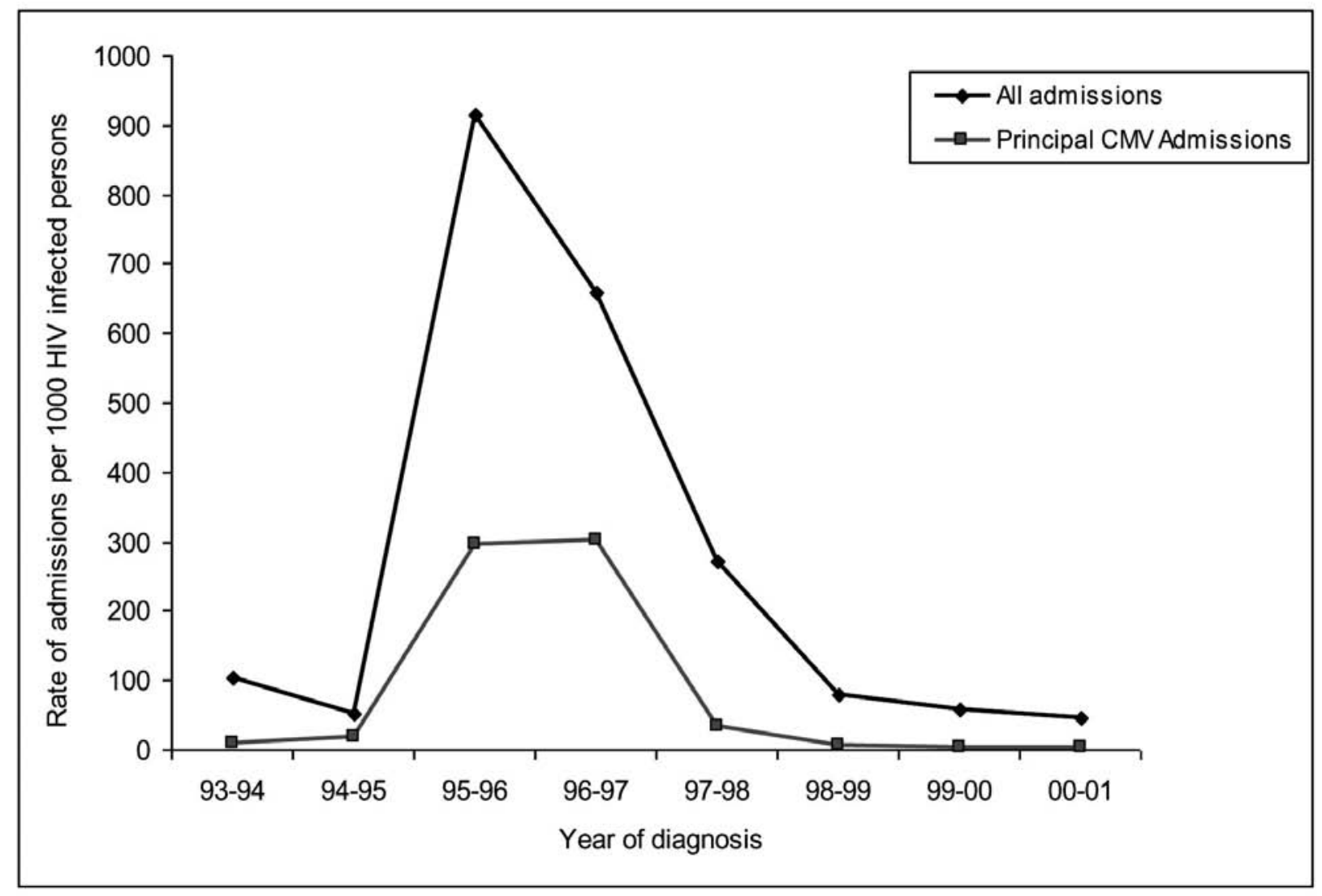

Fig. (1). Rate of hospitalisations for HIV and CMV in Australia, per 1000 HIV-infected population, Australia, July 1993 to June 2001. 
(range: 1-678 days), whilst $76.3 \%$ of admissions were for 48 hours of less. Over the eight year period there were 611 deaths, of which $8.4 \%$ were patients with CMV as their principal diagnosis.

Over the eight-year period, $30.2 \%(6610 / 21846)$ of the HIV infected patients admitted to hospital were principally diagnosed with CMV disease. Over the whole eight years, the average annual rate of these admissions was 83.6 episodes per 1000 population. These types of episodes peaked in 1996-1997 at 300 cases per 1000 HIV population and then declined rapidly to 3.9 cases per $1000 \mathrm{HIV}$ population by 2000-2001.

Of the total admissions recorded for CMV in HIV patients over the eight years, $96.2 \%(21,029)$ were males. The average rate was for all years higher in males (277 episodes per 1000 male HIV-infected population), than in females (183 episodes per 1000 female HIV-infected population) (Fig. 2). In 1993-1994, the hospitalization rate for males was 105 episodes; it then rose to 943 episodes by 1995-1996 and then declined to 45 episodes per 1000 male HIV-infected population by 2000-2001. A Chi-square test for trend indicated a significance association between CMV/HIV admissions for males and HIV-infected male population of Australia for the study period $(n=18657, p=<0.05)$.

As documented in Table 1, the average age-specific rate of CMV in this period was highest for those aged 40-44 years (64.9 episodes per $1000 \mathrm{HIV}$-infected population aged
40-44 years), followed by those aged 35-39 years (55 episodes per $1000 \mathrm{HIV}$-infected population aged 35-39 years) and 45-49 year olds (51.2 episodes per 1000 HIV-infected population aged 45-49 years). There were 22 admissions for children and adolescents under the age of 18 years.

\section{DISCUSSION}

In this study, the annual hospital admissions patterns for CMV in Australia over an eight year period were analyzed. Rates were shown to be extremely high in the periods of 1995-1996 and 1996-1997. By 2000-2001, the rate of hospital admissions was only 44.9 cases per 1000 HIV-infected population

To the best of our knowledge, this is the first study to examine general hospital trends for CMV/HIV-infected patients over the HAART transition. One previous hospital study [19] conducted a retrospective review of all HIV infected patients diagnosed with CMV retinitis in two major hospitals in Melbourne. However, this study was undertaken prior to the introduction of HAART. The authors found that between 1984 and 1996, CMV retinitis was diagnosed in $16.5 \%(212 / 1281)$ hospitalised patients with AIDS. Follow up showed that by June 1998, 193 (93\%) of the participants had died, at a median time of 36 weeks (range 0-192 weeks) from CMV diagnosis. Moderate visual loss was recorded in $35 \%$ of patients, whilst $14 \%$ developed severe visual loss from a diagnosis of CMV retinitis. The authors acknowledge

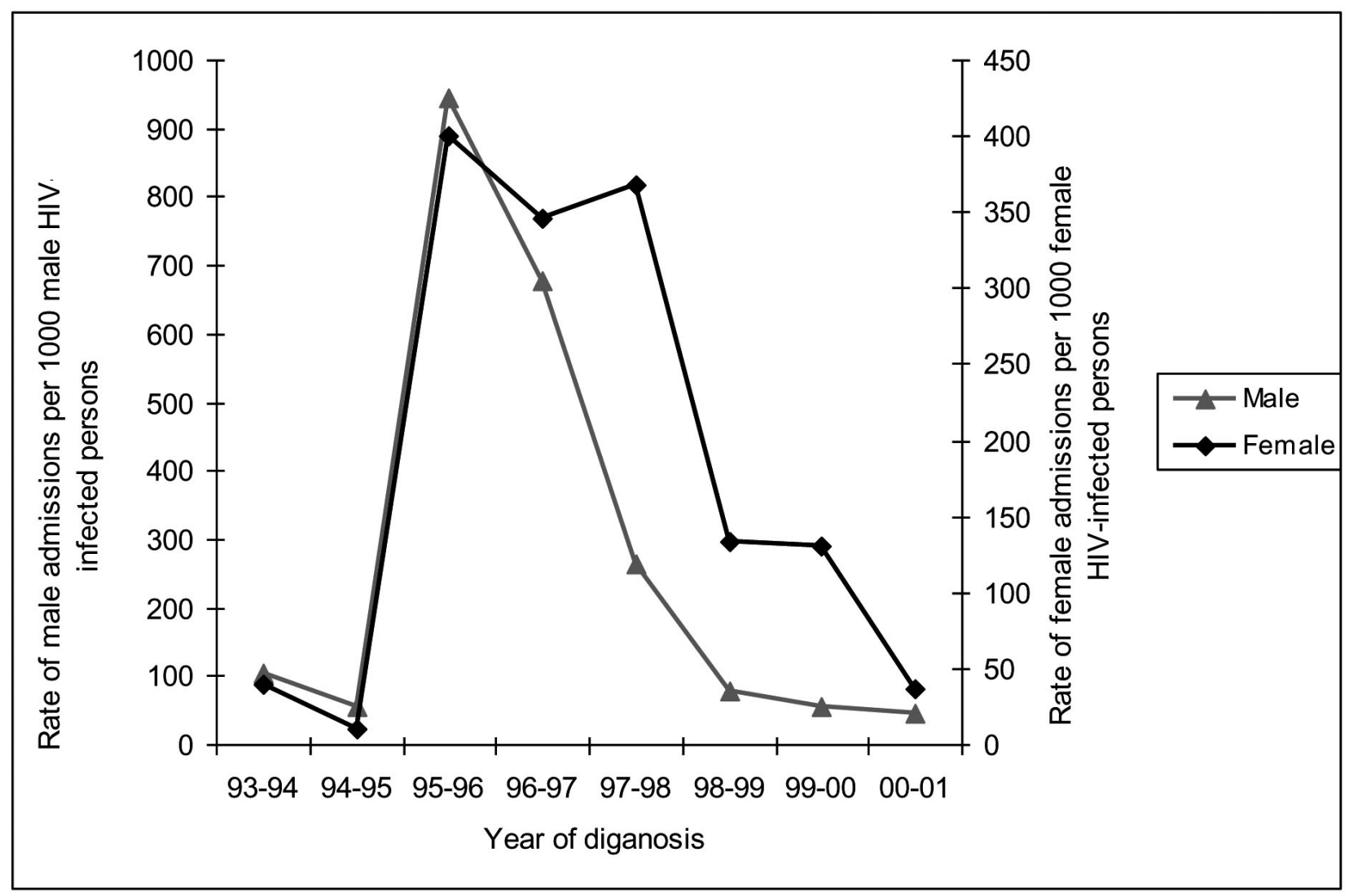

Fig. (2). Rate of hospitalisations for HIV and CMV in Australia, per 1000 HIV-infected population, by sex, Australia, July 1993 to June 2001. 
Table 1. CMV Hospitalisations in HIV Patients by Age Group, Australia, July 1993 to June 2001

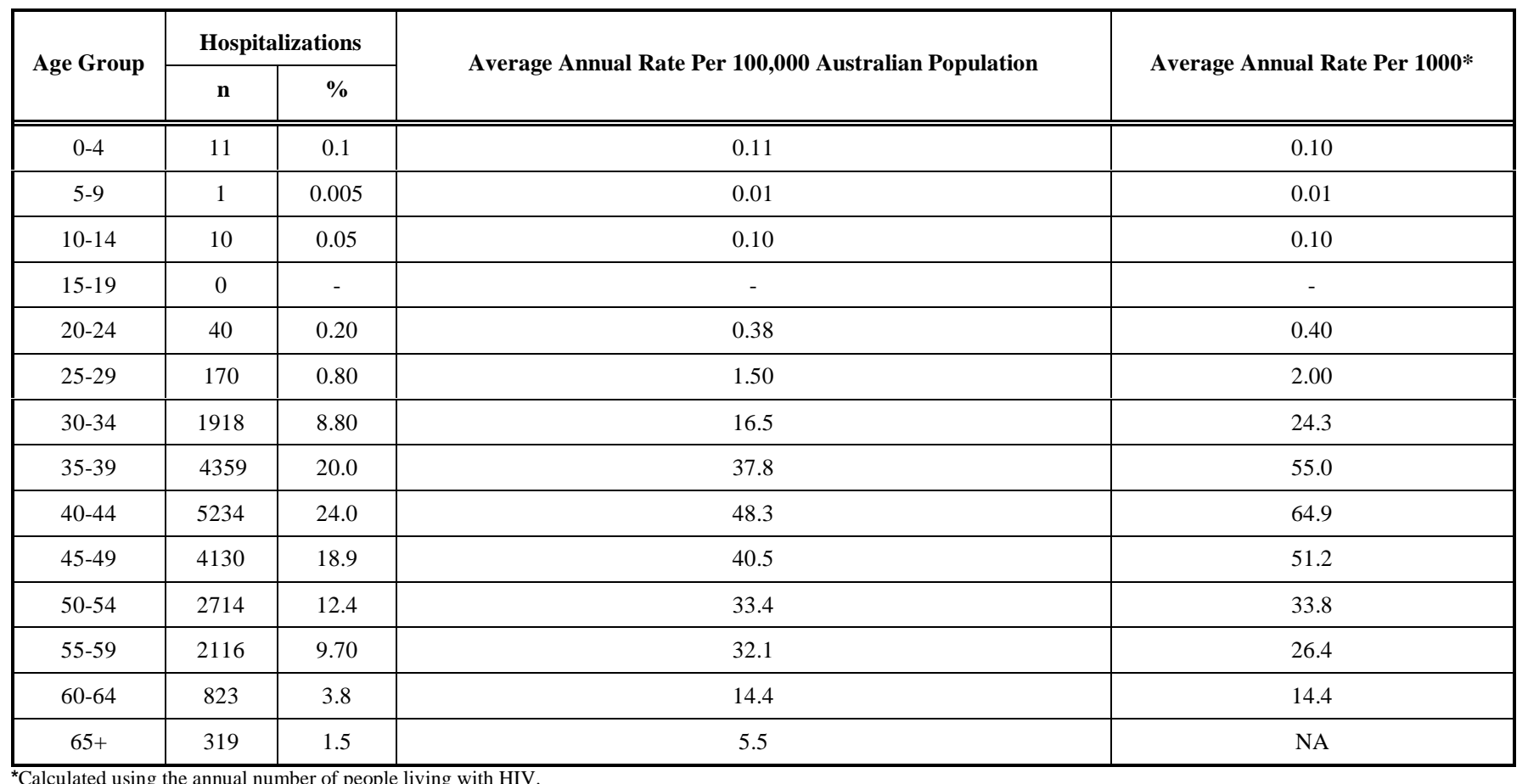

that since the advent of HAART, a reduction has occurred in the incidence of new diagnoses and the survival time of patients with CMV retinitis has prolonged.

Previous studies examining the impact of HAART on the burden of CMV have been based on cross-sectional data. One study reported in their HIV cohort (a geographical defined area in Canada), that the rate of first episode CMV disease declined from a mean of 40 cases per 1000 personyears (during the pre-HAART era) to four cases per 1000 person-years during the HAART era. The authors also reported that both the prevalence of CMV disease in their population fell from $15 \%$ in the pre-HAART era to $4 \%$ with the advent of HAART and the mean mortality rate associated CMV disease decreased from 178 to 27 deaths per 1000 person-years with the use of HAART $(\mathrm{P}<0.05$ by non-pooled $\mathrm{t}$ test) [20]. In corroboration with these findings are the results from a second study, which also reported a decline in the rate of CMV disease from 7.34 (95\% CI: 6.17-8.86) in the preHAART era (1993-1996) to 0.75 (95\% CI: 0.40-1.28) in the HAART era (1997-1999). The authors also reported that survival increased from the pre-HAART to the HAART era [1].

In this study, admissions for CMV and HIV peaked in 1995-1996 and 1996-1997 and then declined. On examination of these cases, we found that over $50 \%$ of patients admitted during this time were diagnosed with a principal diagnosis of CMV, and a secondary code of "HIV-related infection". However, there were no other discerning features to classify these patients. Potential explanations for this peak in admissions include artifacts or errors in the coding, improvements in screening or changes in access, admission practices and record coding. It was also hypothesized that clinical trials examining the use of HAART in these populations might have been running over these two financial period, which may have required participants with HIV to be admitted to hospital. Given the very high number of admissions for less than 48 hours, this might be a reasonable explanation.

After the peak years, admission rates decline rapidly. Presumably, the diffusion of HAART in 1996 led to improvements in clinical status and reduced the need for inpatient HIV care. Unfortunately, due to the limitation of the data, we were unable to ascertain any specific information on use of antiretroviral medications in these patients. The recognition of HAART as the factor accounting for declines in inpatient use is thus based on circumstantial evidence. One study has suggested factors other then HAART contributed to the reduction in CMV/HIV cases, including a decreasing proportion of $\mathrm{HIV}$-infected homosexual men relative to groups with lower CMV seroprevalence, or better prophylaxis of other opportunistic infections that could be cofactors for CMV disease [21].

In the interpretation of our findings, several methodological limitations need to be addressed. Firstly, our inferences about the CMV burden in the hospital setting are affected by the limitations in the methods of the reporting system. In general, hospitalisation data is recorded fairly uniformly, when compared with other health sources [22]. However, as the information used was collected passively, one must be cautious when assessing the trends because of the possibility that variation in access, admission practices and record coding may occur between regions and over time. It must also be noted that the hospitalisation databases contains a record for each admission, which means that there are separate records for each re-admission or inter-hospital transfer.

People with HIV may still present with OIs. HAART does not work for everyone, as many people experience antiretroviral failure, intolerable side effects, or the devel- 
opment of drug-resistant HIV. The advent of HAART has had a remarkable influence on the occurrence and burden of CMV infections.

\section{ACKNOWLEDGEMENTS}

The National Centre for Immunization Research and Surveillance (NCIRS) funded this study. NCIRS is supported by The Australian Government Department of Health and Ageing, the New South Wales Department of Health and The Children's Hospital at Westmead.

\section{REFERENCE}

[1] Salzberger B, Hartmann P, Hanses F, et al. Incidence and prognosis of CMV disease in HIV-infected patients before and after introduction of combination antiretroviral therapy. Infection 2005; 33(5-6): 345-9.

[2] Hoover D. Clinical manifestations of AIDS in the era of pneumocystis prophylaxis. Multicenter AIDS Cohort Study. N Engl J Med 1993; 329: 1922-6.

[3] Moore RD, Chaisson RE. Natural history of opportunistic disease in an HIV-infected urban clinical cohort. Ann Intern Med 1996; 124(7): 633-42.

[4] Finkelstein DM, Williams PL, Molenberghs G, et al. Patterns of opportunistic infections in patients with HIV infection. J Acquir Immune Defic Syndr 1996; 12(1): 38-45.

[5] Drew WL. Cytomegalovirus disease in the highly active antiretroviral therapy era. Curr Infect Dis Report 2003; 5: 257-65.

[6] Palella FJ Jr, Delaney KM, Moorman AC, et al. Declining morbidity and mortality among patients with advanced human immunodeficiency virus infection. HIV Outpatient Study Investigators. N Engl J Med 1998; 338(13): 853-60.

[7] Mocroft A, Sabin CA, Youle M, et al. Changes in AIDS-defining illnesses in a London Clinic, 1987-1998. J AIDS 1999; 21(5): 4017.

[8] Brodt HR, Kamps BS, Gute P, Knupp B, Staszewski S, Helm EB. Changing incidence of AIDS-defining illnesses in the era of antiretroviral combination therapy. AIDS 1997; 11(14): 1731-8.

[9] Casado JL, Arrizabalaga J, Montes M, et al. Incidence and risk factors for developing cytomegalovirus retinitis in HIV-infected patients receiving protease inhibitor therapy. Spanish CMV-AIDS Study Group. AIDS 1999; 13(12): 1497-502.

[10] Verbraak FD, Boom R, Wertheim-van Dillen PM, et al. Influence of highly active antiretroviral therapy on the development of CMV disease in HIV positive patients at high risk for CMV disease. Br J Opthalmol 1999; 83(10): 1186-9.

[11] Salmon-Ceron D, Mazeron MC, Chaput S, et al. Plasma cytomegalovirus DNA, pp65 antigenaemia and a low CD4 cell count remain risk factors for cytomegalovirus disease in patients receiving highly active antiretroviral therapy. AIDS 2000; 14(8): 1041-9.

[12] Labetoulle M, Goujard C, Frau E, et al. Cytomegalovirus retinitis in advanced HIV-infected patients treated with protease inhibitors: incidence and outcome over 2 years. J AIDS 1999; 22(3): 228-34.

[13] Salmon D. CMV DNA pp65 antigenaemia and low CD4 count remain risk factors for CMV disease in patients receiving HAART. J Acquit Immun Defic Syndr 2000; 14: 1041-9.

[14] Mocroft A, Katlama C, Johnson AM, et al. AIDS across Europe, 1994-98: the EuroSIDA study. Lancet 2000; 356(9226): 291-6.

[15] Kestelyn PG, Cunningham ET Jr. HIV/AIDS and blindness. Bull World Health Organ 2001; 79(3): 208-13.

[16] Dore GJ, Li Y, McDonald A, Ree H, Kaldor JM. National HIV Surveillance Committee. Impact of highly active antiretroviral therapy on individual AIDS-defining illness incidence and survival in Australia. J AIDS 2002; 29(4): 388-95.

[17] Australian Institute of Health and Welfare (AIHW). Australian hospital statistics. Canberra: AIHW; 1993-2001.

[18] Kaldor J, McDonald AM, Blumer CE, et al. The acquired immunodeficiency syndrome in Australia: incidence 1982-1991. Med J Aust 1993; 158(1): 10-7.

[19] Cherry CL, Mijch AM, Hoy JF, et al. HIV associated cytomegalovirus retinitis in Melbourne, Australia. Sex Transm Infect 2000; 76(3): 221.

[20] Kim S, Snider JJ, Gill MJ. Cytomegalovirus Disease in HIV Infection: Twenty Years of a Regional Population's Experience. CID 2006; 42: 1808-9.

[21] Guest JL, Rimland D. Decreased incidence of CMV disease unrelated to use of HAART. Abstracts of the 32nd Annual Meeting of The Society For Epidemiologic Research. Baltimore, Maryland: Am J Epidemiol 1999.

[22] McIntyre P, HF G, Gilmour R, et al. Vaccine preventable diseases and vaccination coverage in Australia, 1999 to 2000. Commun Dis Intell 2002; 1: 1-111. 